OPEN ACCESS

Edited by:

Adelaide De Vecchi,

King's College London,

United Kingdom

Reviewed by:

Bradley S. Fleenor,

Ball State University,

United States

Stefano Tarantini,

University of Oklahoma

Health Sciences Center,

United States

*Correspondence:

Ghassan S. Kassab

gkassab@calmi2.org

Specialty section:

This article was submitted to

Vascular Physiology,

a section of the journal

Frontiers in Physiology

Received: 06 March 2020

Accepted: 11 May 2020

Published: 09 June 2020

Citation:

Guo X and Kassab GS (2020) Increased Serum Klotho With Age-Related Aortic Stiffness and Peripheral Vascular Resistance in Young and Middle-Aged Swine.

Front. Physiol. 11:591.

doi: 10.3389/fphys.2020.00591

\section{Increased Serum Klotho With Age-Related Aortic Stiffness and Peripheral Vascular Resistance in Young and Middle-Aged Swine}

\author{
Xiaomei Guo and Ghassan S. Kassab* \\ California Medical Innovations Institute, San Diego, CA, United States
}

The anti-aging function of Klotho gene has been implicated in age-related diseases. The physiological importance of Klotho in the progression of arterial stiffness with aging, however, remains unclear. The goal of this study is to determine the correlation of circulating Klotho with early age-related aortic stiffening and peripheral hemodynamics. We measured serum Klotho levels in a group of pigs with age ranges of 1.5-9 years and investigated the relationship between Klotho levels and biomarkers of aortic stiffening with aging, including aortic pulse wave velocity (PWV), augmentation index (Alx), and pulse pressure (PP). The effects of aortic stiffening on peripheral vascular resistance, compliance, and function were also evaluated. We found that increased aortic stiffness occurred at middle age (>5 years old), as evidenced by an increase in PWV and Alx $(p<0.001)$, but with no changes in blood pressure and PP. With advancing age, increased femoral vascular resistance positively correlated with aortic PWV and Alx $(p<0.01)$. No significant difference in endothelium function and arterial compliance for femoral and small peripheral arteries was observed between young and middle-aged groups. The serum Klotho levels were lower in young and higher in middle-aged pigs $(p<0.001)$, and a positive correlation was found between Klotho and aortic PWV, Alx, and femoral vascular resistance $(p<0.01)$. Our findings suggest that early-aged aortic stiffening has adverse effect on peripheral hemodynamics, independent of blood pressure levels. Elevated Klotho secretion was associated with increased aortic stiffness and peripheral vascular resistance with aging.

Keywords: Klotho, aging, aortic stiffness, vascular resistance, femoral artery

\section{INTRODUCTION}

It is well known that aging is a potent, independent risk factor for cardiovascular disease (Greenwald, 2007; Mitchell et al., 2007). In response to aging, the central elastic arteries become progressively stiffer and their ability to absorb pulsations from the ejecting ventricle is reduced (Avolio et al., 1985; Kelly et al., 1989). Enormous epidemiological research has demonstrated that aortic stiffening increases pulsatile hemodynamic forces, which may exert a detrimental effect on peripheral vascular function, invoking a mechanism linking increased arterial stiffness to the age-related disorders (James et al., 1995; Mitchell et al., 2005). 
Although a marked age-related increase in aortic stiffness has been confirmed in normal subjects before 60 years of age (Mitchell et al., 2004, 2007), it is unclear whether peripheral vascular resistance and function change in response to early aortic aging. Hence, one goal of this study is to determine the relationship between early age-related aortic stiffening and peripheral hemodynamics in young and middle-aged swine.

Klotho gene was originally identified as an anti-aging protein, which encodes a single-pass transmembrane glycoprotein (Kuro-o et al., 1997; Shiraki-Iida et al., 1998). It is produced mainly in the kidney and the soluble form of Klotho is cleaved and released in the blood, urine, and cerebrospinal fluid (Imura et al., 2004; Moe and Drueke, 2008). In a transgenic mouse model, overexpression of Klotho by genetic manipulation of full-length Klotho resulted in a significant extension of life span (Kurosu et al., 2005). The deletion of Klotho gene in mice led to a premature aging syndrome (Kuro-o et al., 1997). In humans, circulating levels of soluble Klotho decrease with age (Xiao et al., 2004), and emerging evidence suggests that Klotho concentration is closely correlated to the development of cardiovascular disease (Semba et al., 2011; Hu et al., 2013) and chronic kidney disease (CKD) (Akimoto et al., 2012; Asai et al., 2012). Although the anti-aging function of Klotho in human aging and age-related disorders has been implicated (Xiao et al., 2004; Hu et al., 2013; Koyama et al., 2015), the biological role of circulating Klotho in the progression of arterial stiffness with the aging process has not yet been elucidated. The second goal of this study is to investigate the associations of serum Klotho with aortic stiffness and peripheral vascular resistance during aging from young to middle age in swine.

In this study, we chose swine as the experimental subject since swine is considered an ideal preclinical model due to the anatomical and physiological similarities in the cardiovascular system between swine and human (Swindle and Smith, 1998). We measured the serum Klotho concentrations in young and middle-aged swine and explored the possible connection between the serum Klotho level and signs of arterial stiffening with aging, including aortic pulse wave velocity (PWV), augmentation index (AIx), and pulse pressure (PP). The effects of early age-related aortic stiffening on peripheral vascular resistance, compliance, and function were also evaluated.

\section{MATERIALS AND METHODS}

\section{Experimental Animals}

A total of 36 female Yucatan miniature pigs were divided into a young group $(n=18)$ with age ranges of $1.5-5$ years and a middle-aged group $(n=18)$ with age ranges of 5.5-9 years (defined as middle age according to a life span of 15 years for Yucatan miniature swine). All animals were fed a standard swine diet (Teklad diet 7037, Envigo, Somerset, NJ) twice daily, and the amount of diet provided was $1 \%$ of the body weight of the pigs. All animal experiments were performed in accordance with national and local ethical guidelines, including the Institute of Laboratory Animal Research guidelines, Public Health Service policy, and the Animal Welfare Act, as approved by the
Institutional Animal Care and Use Committee at California Medical Innovations Institute, San Diego.

\section{Blood Pressure Measurements and Pulse Wave Analysis}

Pigs were fasted for $12 \mathrm{~h}$ prior to surgery and pre-anesthetized with TKX (Telazol $10 \mathrm{mg} / \mathrm{kg}$, Ketamine $5 \mathrm{mg} / \mathrm{kg}$, and Xylazine $5 \mathrm{mg} / \mathrm{kg}$, i.m). Pigs were then anesthetized with 2-3\% isoflurane inhalation. Ventilation with $100 \% \quad \mathrm{O}_{2}$ was provided with a respirator and maintained $\mathrm{PCO}_{2}$ at approximately $35 \mathrm{mmHg}$. Under fluoroscopic guidance (Philips Fluoroscopy System), an introducer sheath (6 Fr) was percutaneously inserted into the jugular vein for administration of $0.9 \%$ saline and heparin (100 IU/kg). A sheath (5 Fr) was used to introduce a catheter into the aortic arch (proximal site) via the carotid artery. Another sheath (5 Fr) was used to introduce a catheter into the abdominal aorta (distal site) via the right femoral artery. Those catheters were connected to a pressure transducer (PowerLab, ADInstruments Inc.). The thoracic (aorta arch) and abdominal aortic blood pressure waveforms were recorded from these two locations simultaneously during the procedure. The femoral blood pressure waveform was also recorded with a catheter placement at the left femoral artery via an introducer sheath (4 Fr).

Pulse wave analysis was applied to determine aortic stiffness. PWV calculation is based on the difference in arrival times of the pressure wave at the proximal (aorta arch) and distal (abdominal aorta) locations. Since the tips of pressure catheters placed on the proximal and distal sites are visible on radiographs, the propagation distance was obtained by measuring the distance between the two catheters. PWV $(\mathrm{m} / \mathrm{s})$ was calculated by dividing the propagation distance by the difference between the two arrival times (transit time). AIx is expressed as the difference between the second and first systolic peaks of central aortic waveform calculated as a percentage of $\mathrm{PP}$.

\section{Femoral Flow Measurement and Analysis (Echocardiography)}

The measurement of femoral blood flow velocity was obtained in all animals using an iE33 duplex ultrasonography (Philips, Andover, MA) equipped with L15-7 transducer. The mean velocity waveforms were recorded continuously for $6 \mathrm{~s}$ for further analysis. The diameter of the femoral artery was also recorded by B-mode imaging. According to the flow velocity waveforms, femoral flow volume $(\mathrm{ml} / \mathrm{min})$ was calculated from the time-averaged mean flow velocity and the arterial diameter. Femoral vascular resistance $(\mathrm{mmHg} / \mathrm{ml} / \mathrm{min})$ was calculated by dividing the mean arterial pressure by the flow volume. After flow measurement, the femoral artery and a small peripheral artery (muscular branch of femoral artery with diameter $300-500 \mu \mathrm{m}$ ) were harvested for endothelial function and mechanical testing. Animal was then euthanized with a saturated solution of potassium chloride injection through the jugular vein to arrest the heart under deep anesthesia. 


\section{Endothelial Function}

An isovolumic myograph was used to evaluate the endotheliumdependent vasorelaxation (Lu et al., 2011). Briefly, the segments from femoral and small peripheral arteries were cannulated on both ends in a physiological bath with HEPES physiologic saline solution (HEPES-PSS, concentration in mmol/l: 142 $\mathrm{NaCl}, 4.7 \mathrm{KCl}, 2.7$ sodium HEPES, 3 HEPES acid, 0.15 $\mathrm{NaHPO}_{4}, 1.17 \mathrm{MgSO}_{4}, 2.79 \mathrm{CaCl}_{2}$, and 5.5 glucose, solution gassed by $95 \% \mathrm{O}_{2}$ plus $5 \% \mathrm{CO}_{2}$ ). Both vessels segments were stretched to in situ length and preloaded at the physiological pressure of $80 \mathrm{mmHg}$. The pressure and external diameter were measured with a pressure transducer (Mikro-Tip SPR-524; Millar Instruments) and a digital diameter tracking (DiamTrak v3+; Australia), respectively. The vessel segment was pre-constricted with phenylephrine (PE) by a series of doses $\left(10^{-10}-10^{-5} \mathrm{~mol} / \mathrm{L}\right.$ in the PSS), and then relaxed with acetylcholine (ACh) by a series of doses: $10^{-10}-10^{-5} \mathrm{~mol} / \mathrm{L}$. The endotheliumindependent relaxation to sodium nitroprusside (SNP, $10^{-5} \mathrm{~mol} / \mathrm{L}$ ) was measured to verify the sensitivity of vascular smooth muscle to nitric oxide (NO). The overall contractility of vessel was tested with potassium chloride $(\mathrm{KCl})$ at $60 \mathrm{mmol} / \mathrm{L}$. Contraction was expressed as percentage of the response to $\mathrm{KCl}$. Relaxation was expressed as percentage of pre-contraction to $\mathrm{PE}$.

\section{Mechanical Tests}

The femoral and small peripheral arterial segments were cannulated on both ends and fully relaxed in $\mathrm{Ca}^{2+}$ free HEPES-PSS. The arterial segment was preconditioned with five cyclic changes in pressure from 0 to $180 \mathrm{mmHg}$. The pressure was then increased in $20 \mathrm{mmHg}$ step increments from 20 to $180 \mathrm{mmHg}$ in a staircase manner. The passive pressure (P)-diameter (D) relation was recorded. The lumen crosssectional area (CSA) was computed from the lumen diameter (D), as CSA $=\pi \mathrm{D}^{2} / 4$. The area compliance $\left(\mathrm{C}_{\mathrm{CSA}}\right)$ of the artery was determined by the slope of the pressure-CSA relationship, i.e., $\mathrm{C}_{\mathrm{V}}=\Delta \mathrm{CSA} / \Delta \mathrm{P}$ as described in a previous publication (Guo et al., 2014).

\section{Serum Klotho Level}

Blood samples were collected in ethylenediaminetetraacetic acid (EDTA) tubes for all experimental pigs. After centrifugation at $5,000 \mathrm{rpm}$ and $4^{\circ} \mathrm{C}$ for $15 \mathrm{~min}$, serum was immediately separated and stored at $-80^{\circ} \mathrm{C}$ until analysis. The circulating level of Klotho in the serum was measured by a Klotho ELISA kit (IBL Co. Ltd., Minneapolis, MN) according to the manufacturer's guideline.

\section{Statistical Analysis}

Results were shown as mean \pm standard error of mean (mean \pm SEM). Statistical analysis was performed using SigmaStat (Systat Software, California, USA). The significance of the differences between young and middle-age groups was evaluated by either $t$-test or one-way ANOVA. Multiple linear regression analysis was used to estimate the correlations between age and the study variables. Spearman's correlation test was used to estimate the correlations between Klotho level and the study variables. Significant differences between the dose-dependent groups for endothelial function testing were determined by two-way ANOVA. The results were considered statistically significant when $p<0.05$ (2-tailed).

\section{RESULTS}

The mean age, body weight, hemodynamic parameters, and serum Klotho level were presented and compared between young and middle-aged groups in Table 1. The mean age is $3.5 \pm 0.3$ years (range of $1.5-5$ years) for the young group and $7.2 \pm 0.3$ years (range of 5.5-9 years) for the middle-aged group. The two study groups have comparable values of body weight and heart rate. There was no between-groups difference in PP, systolic blood pressure (SBP), diastolic blood pressure (DBP), and mean arterial pressure (MAP) in thoracic and abdominal aortic pressure measures. Figure 1 shows a positive correlation of aortic PWV and AIx with age $(p<0.001)$ by using linear regression analysis. Aortic PWV and AIx was significantly increased in middle-aged group as compared to young group $(p<0.001)$. No correlation was found between blood pressure and PP with age (data not shown).

There was no significant difference in PP, SBP, DBP, and MAP in the femoral pressure measures between young and middle-aged groups (Table 1). No difference in diameter of

TABLE 1 | Comparison of age, weight, hemodynamic parameters and serum Klotho levels between young and middle-aged pigs.

\begin{tabular}{|c|c|c|c|}
\hline Variables & $\begin{array}{l}\text { Young } \\
(n=18)\end{array}$ & $\begin{array}{l}\text { Middle-aged } \\
\quad(n=18)\end{array}$ & $p$ \\
\hline Age (years) & $3.5 \pm 0.3$ & $7.2 \pm 0.3$ & $<0.001$ \\
\hline Body weight (kg) & $112.8 \pm 3.3$ & $116.3 \pm 3.0$ & 0.415 \\
\hline Heart rate (beats/min) & $74.8 \pm 2.5$ & $71.3 \pm 2.3$ & 0.31 \\
\hline \multicolumn{4}{|c|}{ Thoracic aortic blood pressure $(\mathrm{mmHg})$} \\
\hline Mean pressure & $81.3 \pm 2.7$ & $77.0 \pm 4.2$ & 0.369 \\
\hline Systolic pressure & $101.1 \pm 2.6$ & $97.6 \pm 4.5$ & 0.475 \\
\hline Diastolic pressure & $71.3 \pm 2.9$ & $66.8 \pm 4.1$ & 0.333 \\
\hline Pulse pressure & $29.7 \pm 1.5$ & $30.8 \pm 1.4$ & 0.592 \\
\hline \multicolumn{4}{|c|}{ Abdominal aortic blood pressure $(\mathrm{mmHg})$} \\
\hline Mean pressure & $78.7 \pm 2.6$ & $75.0 \pm 4.1$ & 0.411 \\
\hline Systolic pressure & $105.8 \pm 2.9$ & $101.1 \pm 4.9$ & 0.372 \\
\hline Diastolic pressure & $66.1 \pm 2.7$ & $62.9 \pm 3.9$ & 0.36 \\
\hline Pulse pressure & $39.7 \pm 2.0$ & $38.6 \pm 2.4$ & 0.805 \\
\hline Aortic PWV (m/s) & $5.4 \pm 0.1$ & $7.7 \pm 0.3$ & $<0.001$ \\
\hline Aortic Alx (\%) & $12.8 \pm 1.2$ & $21.7 \pm 1.1$ & $<0.001$ \\
\hline \multicolumn{4}{|l|}{ Femoral blood pressure $(\mathrm{mmHg})$} \\
\hline Mean pressure & $70.8 \pm 3.0$ & $72.8 \pm 3.7$ & 0.648 \\
\hline Systolic pressure & $90.6 \pm 4.3$ & $92.7 \pm 5.1$ & 0.741 \\
\hline Diastolic pressure & $60.8 \pm 2.5$ & $61.1 \pm 3.6$ & 0.952 \\
\hline Pulse pressure & $29.8 \pm 2.2$ & $31.6 \pm 2.4$ & 0.559 \\
\hline Femoral artery diameter (mm) & $4.02 \pm 0.1$ & $4.07 \pm 0.5$ & 0.727 \\
\hline Femoral mean flow rate (ml/min) & $394.2 \pm 21.1$ & $277.1 \pm 16.2$ & $<0.001$ \\
\hline $\begin{array}{l}\text { Femoral vascular resistance } \\
(\mathrm{mmHg} / \mathrm{ml} / \mathrm{min})\end{array}$ & $0.19 \pm 0.01$ & $0.27 \pm 0.017$ & 0.002 \\
\hline Serum Klotho levels (pg/ml) & $809.8 \pm 193.6$ & $2158.4 \pm 164.5$ & $<0.001$ \\
\hline
\end{tabular}

Values are mean \pm SEM

Bold values are presented when $p<0.05$. 


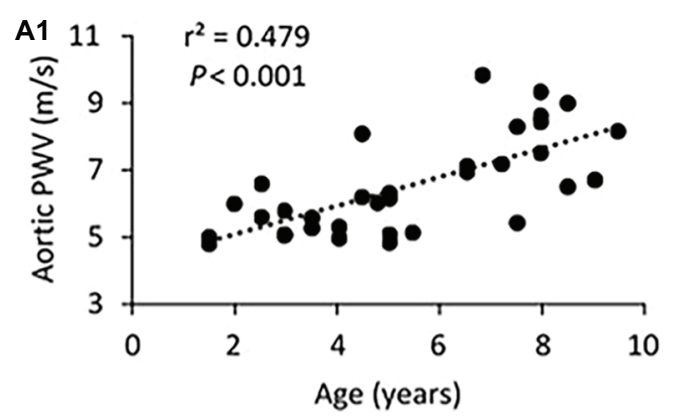

B1

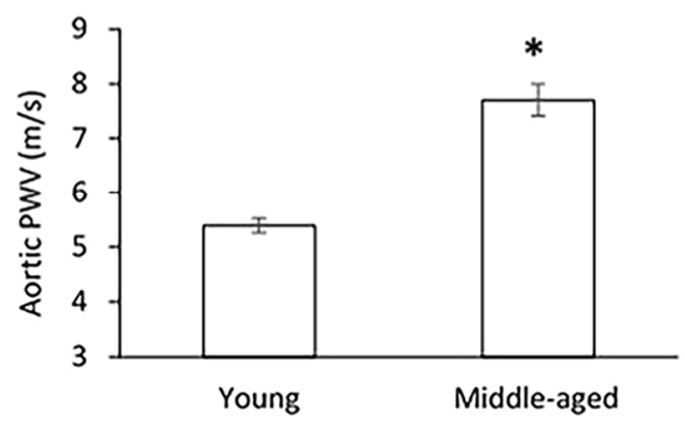

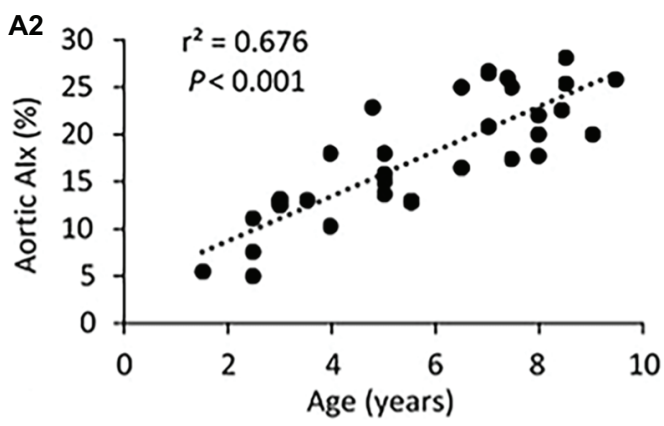

B2

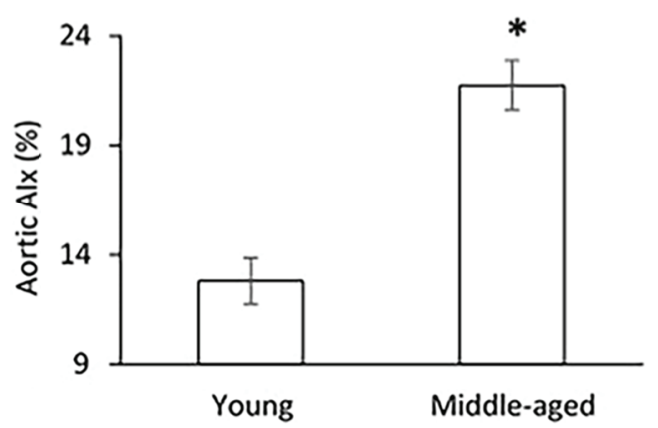

FIGURE 1 | (A) Correlations of aortic pulse wave velocity (PWV) (A1) and augmentation index (Alx) (A2) with age. The linear regression analysis was conducted, and data are expressed as correlation coefficients and significance levels $\left(r^{2} ; p\right)$. (B) Comparison of aortic PWV (B1) and Alx (B2) between young and middle-aged pigs. Data corresponds to means \pm standard error of mean (mean \pm SEM). $p<0.05$, when compared to young group.

femoral artery was seen between these two groups. Figure 2 shows the correlation between vascular resistance and mean flow of femoral artery with age. The femoral vascular resistance was significantly increased $(p<0.01)$, whereas mean flow was significantly decreased $(p<0.01)$ in middle-aged as compared to young group. A linear relationship was observed between femoral vascular resistance and mean flow with age $(p<0.001)$. With regards to aortic stiffness, aortic PWV, and AIx were positively correlated with femoral vascular resistance $(p<0.01)$ and negatively correlated with femoral mean flow $(p<0.01)$ as shown in Figure 3.

Compliance of arteries was presented as the pressure-crosssectional area (P-CSA) relationship of the femoral artery and small peripheral artery. Although the middle-aged group has lower values of CSA compliance in both femoral and small peripheral arteries as compared to the young group (Figure 4), the differences are not statistically significant.

Figure 5A shows the correlation between serum Klotho level and age. The mean Klotho level of the middle-aged group was significantly higher than that of the young-aged group $(2,158.4 \pm 164.5$ vs. $809.8 \pm 193.6 \mathrm{pg} / \mathrm{ml}, p<0.001)$. The Spearman's correlation test confirms that the serum Klotho level was positively correlated with age within the age ranges of $1.5-9$ years $(p<0.001)$. There was a significantly positive correlation between serum Klotho levels and PWV $(p<0.001)$ and AIx $(p=0.001)$ as shown in Figure 5B. In Figure 5C, the Klotho level was also positively associated with femoral vascular resistance $(p<0.001)$, but slightly inversely correlated with femoral flow
( $p=0.005)$. There was no apparent correlation of Klotho levels with aortic and femoral blood pressure and PP (data not shown).

Vascular endothelial function was evaluated by ex-vivo $\mathrm{PE}$ pre-contractile endothelium-dependent vasorelaxation. No significant difference in the vascular contractions to PE for both femoral and small peripheral arteries was observed between young and middle-age groups as shown in Figure 6A. For the femoral artery segment $(4.04 \pm 0.3 \mathrm{~mm}$ in diameter), the endothelium-dependent vasodilation in response to ACh was similar in middle-age groups compared with young group (Figure 6B1). For the small peripheral artery $(410 \pm 30 \mu \mathrm{m}$ in diameter), the vasodilation to ACh in middle-age group tended to decrease, but the change was not significantly different from the young group ( $p=0.08$, Figure 6B2). In middle-aged group, the vascular contraction to $\mathrm{KCl}$ at $60 \mathrm{mmol} / \mathrm{L}$ did not show significant difference as compared to the young group for both femoral and small peripheral arteries (Figure 6C1). There was no significant difference in the maximal responses of endotheliumindependent vasodilation to SNP at $10^{-5} \mathrm{~mol} / \mathrm{L}$ between young and middle-aged groups for both arteries (Figure 6C2).

\section{DISCUSSION}

To our knowledge, this is the first study of the relation between Klotho and aortic stiffness and associated peripheral hemodynamics in swine. The major findings are as follows: (1) aortic PWV and AIx had a significant positive correlation 


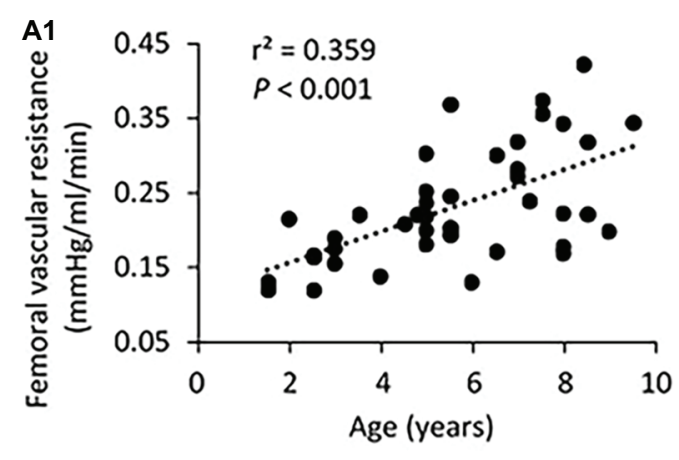

B1

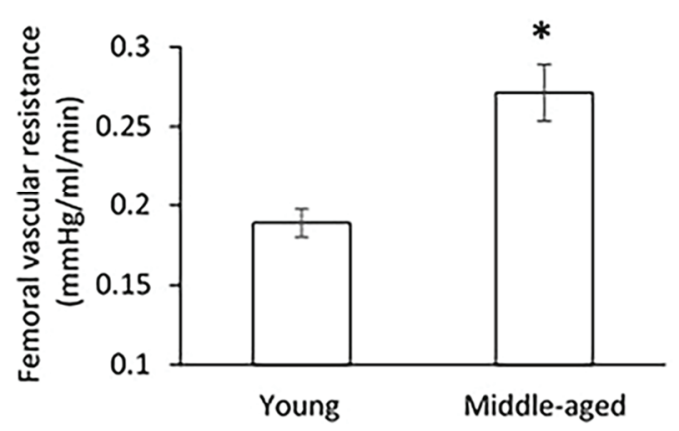

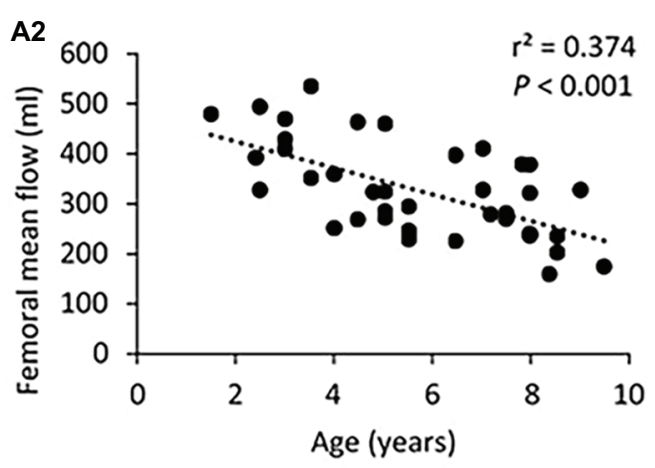

B2

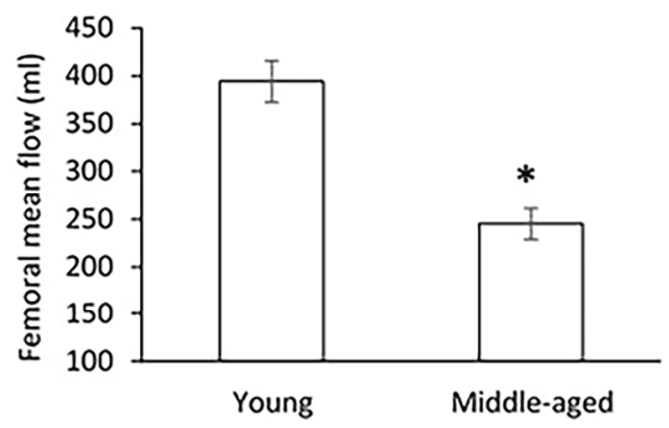

FIGURE 2 | (A) Correlations of femoral vascular resistance (A1) and mean flow (A2) with age. The linear regression analysis was conducted, and data are expressed as correlation coefficients and significance levels $\left(r^{2} ; p\right)$. (B) Comparison of femoral vascular resistance (B1) and mean flow (B2) between young and middle-aged pigs. Data corresponds to means \pm SEM. $p<0.05$, when compared to young group.
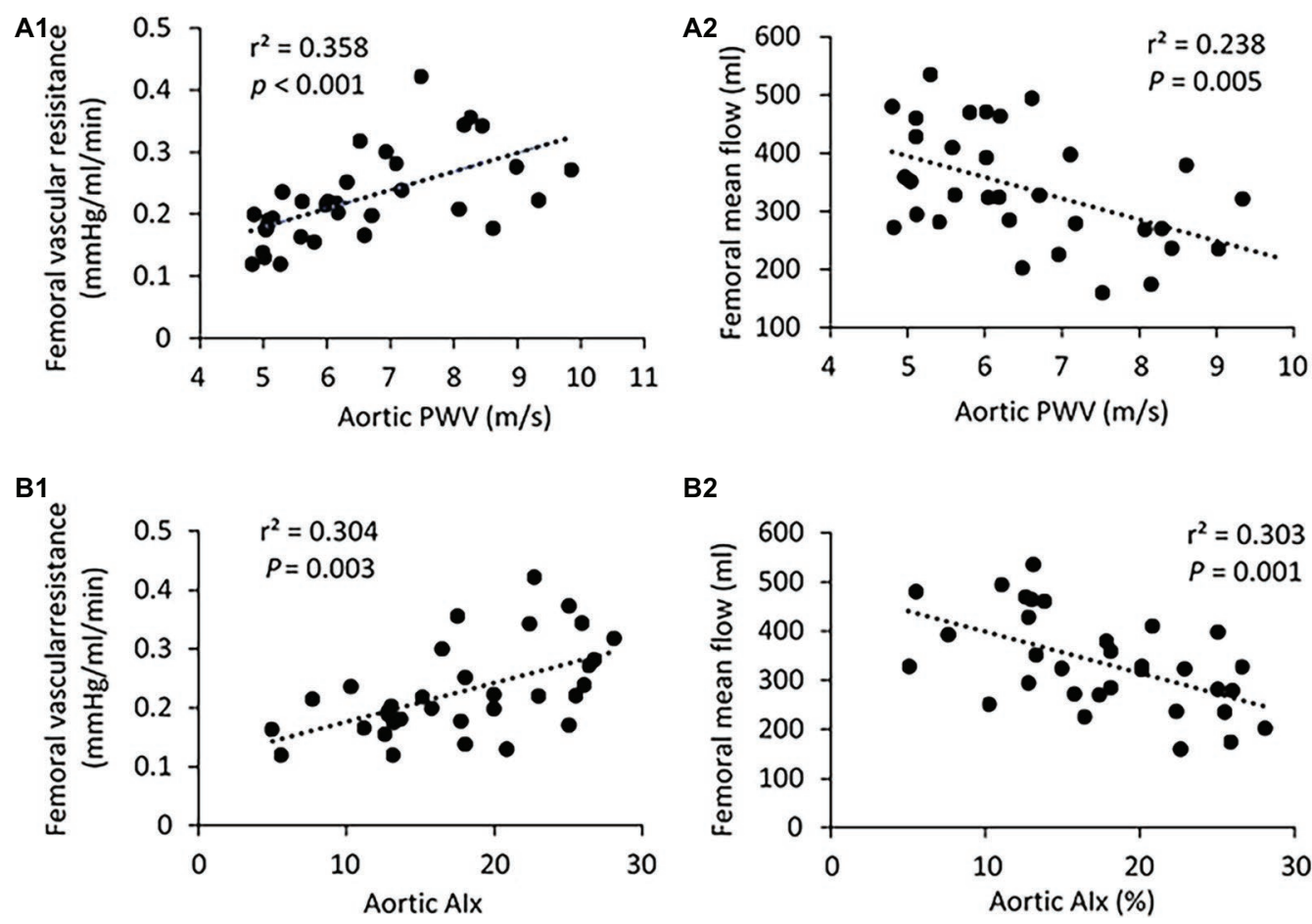

FIGURE 3 | (A) Correlation of aortic PWV with femoral vascular resisitance (A1) and mean flow (A2). (B) Correlation of aortic Alx with femoral vascular resisitance (B1) and mean flow (B2). The linear regression analysis was conducted, and data are expressed as correlation coefficients and significance levels $\left(r^{2} ; p\right)$. 


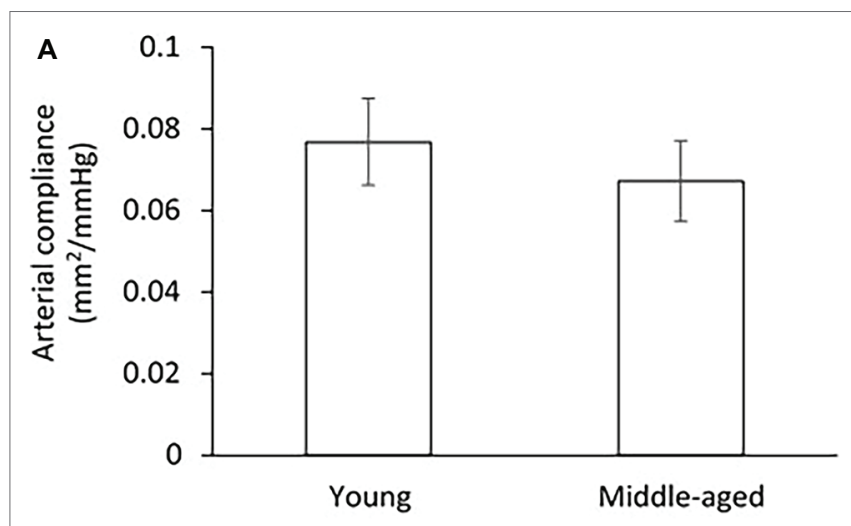

B

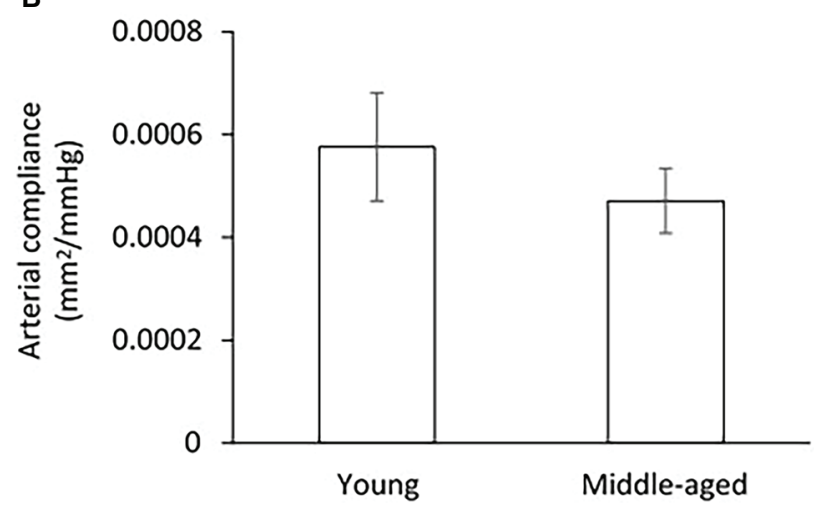

FIGURE 4 | Comparison of arterial compliance for femoral artery (A) and small peripheral artery (B) between young and middle-aged pigs. Data corresponds to means \pm SEM.

with age in a group of pigs 1.5-9 years old; (2) mean aortic and femoral blood pressure, and PP did not differ between young ( $\leq 5$ years) and middle age ( $>5$ years) groups; (3) with advancing age, increased femoral vascular resistance, and decreased femoral flow were associated with aortic stiffening but with no changes in femoral endothelium function and arterial compliance; and (4) serum Klotho levels were lower in young and higher in middle-aged pigs, and positively correlated with aortic PWV, AI, and femoral vascular resistance.

Age-related arterial stiffening has emerged as an independent risk factor for cardiovascular events, including hypertension (Izzo, 2004), myocardial infarction (Mattace-Raso et al., 2006), diabetes (Cruickshank et al., 2002), heart failure (Chae et al., 1999), and stroke (Laurent et al., 2003). There is substantial evidence that aortic PWV and AIx predict cardiovascular morbidity and mortality in humans (Vlachopoulos et al., 2010). PWV represents the speed of a pressure wave propagating down a blood vessel, whereas AIx is a measure of the contribution made by the reflected pressure wave to the ascending aortic pressure waveform; both of which provide a measure of systemic arterial stiffness (Safar and London, 2000; Cecelja and Chowienczyk, 2012). Here, we found that aortic PWV and AIx significantly increased with age in 1.5-9 years old pigs. It has been extensively documented that arterial stiffness increases throughout the normal human life span (Mitchell et al., 2007) and is a major determinant of increased systolic and pulse pressure with advancing age (Kelly et al., 1989; Dernellis and Panaretou, 2005). In the middle-aged pigs, however, PP and SBP were not increased when compared with the younger pigs despite an increase in aortic stiffness. This result is consistent with some clinical observations that most of the age-related increase in PP occurs in elderly individuals (age $>60$ years) and not in younger populations (Burt et al., 1995; Franklin et al., 1999). Although our data demonstrated that aging exerts a negative influence on aortic stiffness (raised PWV and AIx), even at middle age in healthy swine, whether age-related aortic stiffening precedes the development of elevated PP and/or SBP in elderly swine ( $>9$ years old) is not known. This is a limitation of the current study because no swine older than 9 years were available from the vendors during the period of the experiment.

To answer the question of whether early aortic aging affects the hemodynamics of peripheral vasculature, we measured the vascular resistance, mean flow, and arterial compliance of femoral artery (average of $4.04 \pm 0.3 \mathrm{~mm}$ in diameter range $3.2-4.8 \mathrm{~mm}$ ) for all experimental pigs. We also measured the arterial compliance of small peripheral artery (average of $410 \pm 30 \mu \mathrm{m}$ in diameter range 308-520 $\mu \mathrm{m}$ ). In a previous large community-based population study, without established hypertension or advanced vascular disease, a modest rise in forearm vascular resistance was reported to be related to increased aortic stiffness (Mitchell et al., 2005). Here, we found a marked increase in femoral vascular resistance with advancing age. It is reasonable to speculate that increasing peripheral vascular resistance in response to aortic stiffening would functionally reduce local flow, which was exhibited in middle-aged group as compared to young group by the use of ultrasonography measurements. To assess the relations between aortic stiffness and peripheral hemodynamics, we found that the femoral vascular resistance was positively correlated with the aortic PWV and AIx, indicating that early age-related aortic stiffening, even at normotensive PP or blood pressure levels, may have adverse influences on peripheral hemodynamics. In addition, arterial compliance, defined as the changes in luminal dimension (CSA) divided by the corresponding change in pressure, is conventionally thought to be a surrogate marker of arterial elasticity (Cohn et al., 2004). Our data showed no obvious changes in femoral and small peripheral arterial compliance between young and middle-aged groups, meaning a sustained peripheral arterial elasticity in ages $1.5-9$ years of pigs. Similar results have been obtained in Framingham Heart Studies that the compliance or distensibility of large muscular arteries (i.e., femoral) is maintained at normal levels or changed relatively little despite an accelerated aortic stiffness with age (van der Heijden-Spek et al., 2000; Mitchell et al., 2004).

Dysfunction of the vascular endothelium has been associated with high blood pressure and arterial stiffening (Puddu et al., 2000; Safar et al., 2001). We examined the endothelium of femoral artery and found no evidence of endothelial dysfunction since comparable endothelium-dependent vasodilation to $\mathrm{ACh}$ was shown between young and middle-aged groups. 

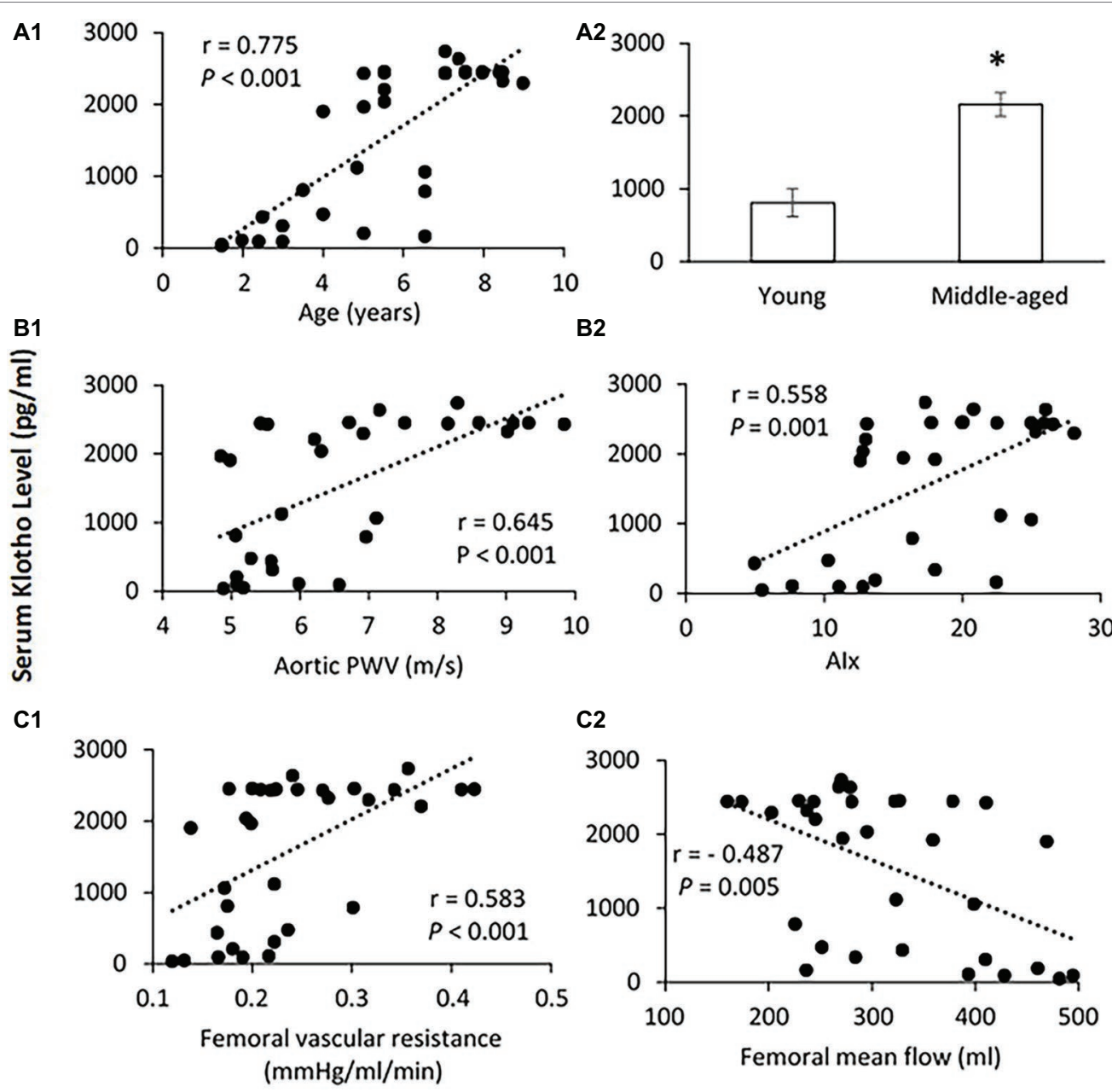

B2

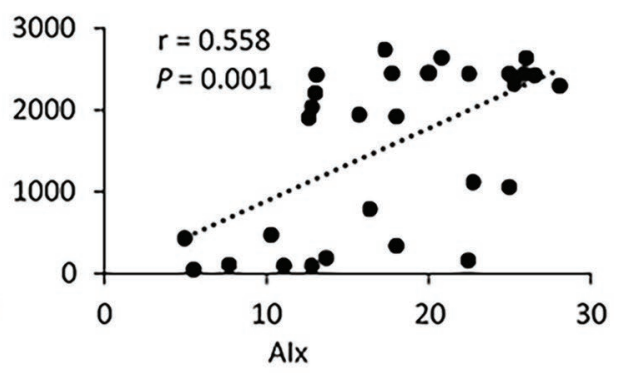

C2

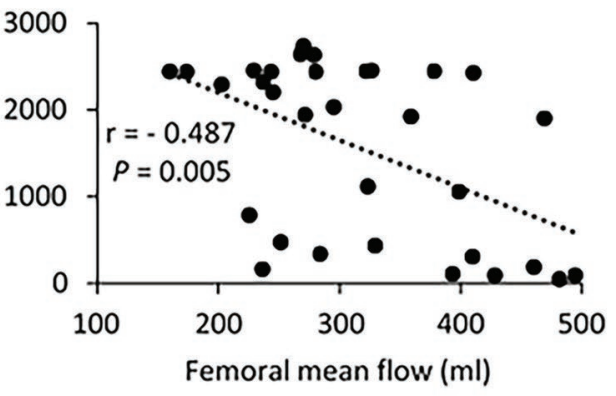

FIGURE 5 | (A) Correlation of serum Klotho level with age (A1) and the mean levels of serum Klotho (A2) in young and middle-aged pigs. Data corresponds to means \pm SEM. $p<0.05$, when compared to young group. (B) Correlations of serum Klotho levels with aortic PWV (B1) and Alx (B2). (C) Correlations of serum Klotho levels with femoral vascular resistance (C1) and mean flow (C2). The Spearman's correlation test was conducted, and data are expressed as correlation coefficients and significance levels $(r ; p)$.

For the small peripheral artery, although the vasodilation to ACh in middle-aged group has a descending trend, the change is not statistically significant compared with the young group $(p=0.08)$. There are emerging data that aortic stiffening may trigger remodeling, rarefaction, or hypertrophy in the microcirculation, leading to increased peripheral vascular resistance to blood flow (James et al., 1995; Liao et al., 2004). Several studies have provided direct evidence that functional and structural impairment of cerebral microcirculation are present in age-related cognitive decline (Toth et al., 2017) and Alzheimer's disease (Csiszar et al., 2017). The current study only exhibited a downward trend in vascular function of peripheral muscular arteries with diameter $>300 \mu \mathrm{m}$, we do not know whether abnormalities in microvasculature $(<150 \mu \mathrm{m}$ in diameter; Levy et al., 2001) occurs during the early-aged aortic stiffening, and whether microvascular dysfunction contributes to the increased peripheral vascular resistance in middle-aged swine. The mechanisms implicating the microcirculation need to be addressed in future studies.
Klotho is an aging suppressor gene whose protective properties are critical for a proper function of many tissues and organs (Kuro-o et al., 1997; Hu et al., 2013). A recent study by Semba et al. (2011) suggested that raised levels of Klotho were related to the lower prevalence of cardiovascular disease. More clinical research has reported that a deficiency of Klotho may be an early biomarker for CKD (Akimoto et al., 2012; Asai et al., 2012) and acute kidney injury (Hu et al., 2010). In this study, surprisingly, we found that the serum Klotho level was significantly increased in middle-aged group as compared to young group. Moreover, a positive relationship between Klotho level and age was seen in ages from 1.5 to 9 years of pigs. This result is contrary to the findings from human studies that circulating Klotho concentrations decreased remarkably with age (range of 0.1-88 years; Xiao et al., 2004; Yamazaki et al., 2010). Recently, Klotho protein was found to be expressed in human aorta (Lim et al., 2012) and coronary artery (Richter et al., 2016), which protects against endothelial dysfunction by increasing 


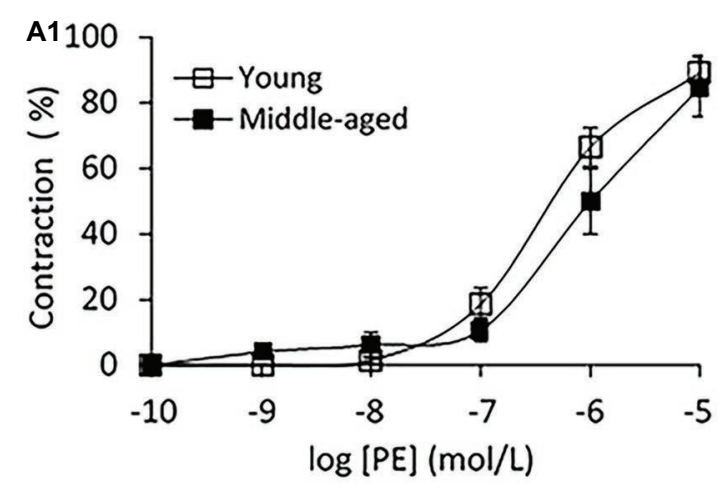

B1
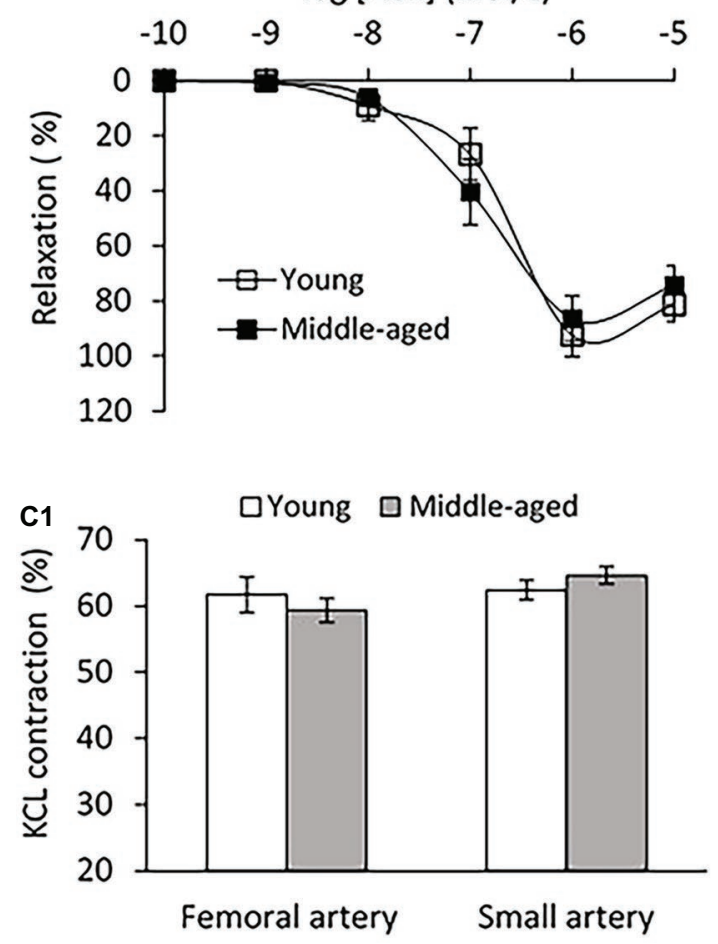

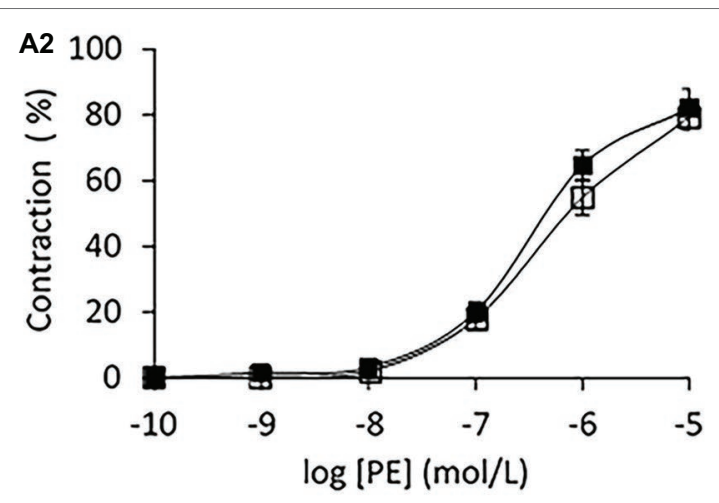

B2
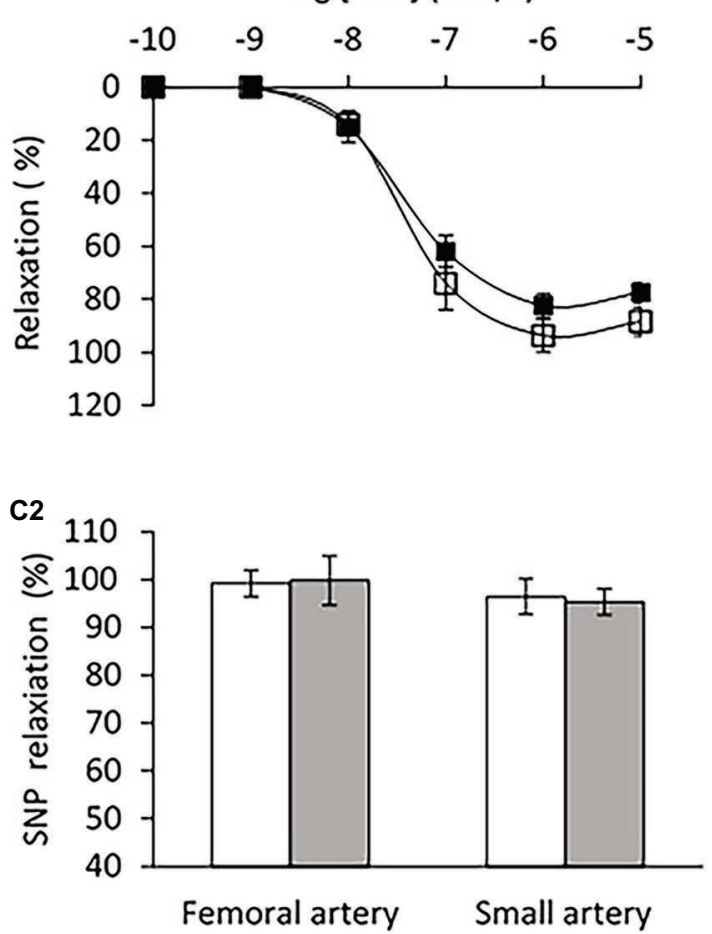

FIGURE 6 | (A) Vascular contraction to phenylephrine (PE) in femoral artery (A1) and small peripheral artery (A2) between young and middle-aged pigs. (B) PE pre-contractile endothelium-dependent vasodilation to acetylcholine (ACh) in femoral artery (B1) and small peripheral artery (B2) between young and middle-aged pigs. (C) Vascular contraction to potassium chloride (KCL) at $60 \mathrm{mmol} / \mathrm{L}$ (C1) and maximal response of vasodilation to sodium nitroprusside (SNP) at $10^{-5} \mathrm{~mol} / \mathrm{L}$ (C2) between young and middle-aged pigs in femoral and small peripheral artery. Data corresponds to means \pm SEM.

nitric oxide production (Saito et al., 1998, 2000). Moreover, the supplementation of exogenous Klotho attenuates oxidative stress, inflammation, and fibrosis by the inhibition of insulin/ insulin-like growth factor-1 (IGF-1) and transforming growth factor-1 (TGF-1) signaling pathways to protect the vasculature and cardiac tissue (Yamamoto et al., 2005; Dalton et al., 2017; Takenaka et al., 2017). It is possible that an increase in serum Klotho level at middle age could be a compensatory response to the increased aortic stiffness with age to maintain endothelium function and arterial compliance of peripheral arterial system. Similar adaptive mechanism has been proposed by Paula et al. (2016) who observed an augmented level of Klotho in patients with myocardial infarction to ameliorate cardiac hypertrophy and remodeling. To the best of our knowledge, this study is the first to measure circulating Klotho concentrations with aging in swine, where species variations could be a potential factor that accounts for the different response of Klotho to advancing age between human and swine. The Spearman's correlation analysis of our data showed that serum Klotho level was positively correlated with aortic PWV, AIx, and femoral vascular resistance, but not with blood pressure and PP. Our results confirmed a positive correlation of serum Klotho levels with age in young to middle-aged swine. Completion of further studies with the involvement of elderly swine ( $>9$ years) is necessary to explore the pathophysiological mechanisms of serum Klotho fluctuation with advancing age. 
Whether Klotho gene provides vascular protection via upregulated Klotho production during early aortic aging remains to be determined as well.

There are limitations in the present study. Several clinical studies have reported that female is more susceptible to age-related cardiovascular events compared with male (Merz and Cheng, 2016; Rodgers et al., 2019). Here, we only focused on female swine with a range in ages 1.5-9 years. We did not evaluate sex variation in changes of serum Klotho and aortic stiffness with aging since no male swine $>2$ years old (and no female $>9$ years) were available from the vendors. It is recognized that different blood- and/or tissue-based biomarkers such as interleukin-6 (IL-6), tumor necrosis factor $\alpha$ (TNF $\alpha$ ), and insulin-like growth factor 1 (IGF-1) are involved in vascular changes during aging (Tarantini et al., 2016; Justice et al., 2018). Most recently, a geroscience-guided clinical trial has suggested that the systematic evaluation of multi-biomarkers may be the best approach to clarify the biologic hallmarks of aging in human research (Justice et al., 2018). The current study, however, solely assessed a single blood-based biomarker "Klotho" which may not be able to reflect the complex and multifactorial processes underlying aging. In addition, we did not directly test the effect of serum Klotho on the aortic stiffness and peripheral vascular resistance. Hence, we cannot conclude any cause-and-effect relation. Our findings indicate an association between circulating Klotho and early age-related aortic stiffening and peripheral hemodynamics in swine.

In summary, early age-related aortic stiffening, even at normotensive PP or blood pressure levels, leads to functional alterations in peripheral hemodynamics, evidenced by increased femoral vascular resistance and reduced local flow. Elevated Klotho secretion with aging is associated with an increase in aortic stiffness and peripheral vascular resistance.

\section{REFERENCES}

Akimoto, T., Shiizaki, K., Sugase, T., Watanabe, Y., Yoshizawa, H., Otani, N., et al. (2012). The relationship between the soluble Klotho protein and the residual renal function among peritoneal dialysis patients. Clin. Exp. Nephrol. 16, 442-447. doi: 10.1007/s10157-011-0582-2

Asai, O., Nakatani, K., Tanaka, T., Sakan, H., Imura, A., Tanaka, T., et al. (2012). Decreased renal a-Klotho expression in early diabetic nephropathy in humans and mice and its possible role in urinary calcium excretion. Kidney Int. 81, 539-547. doi: 10.1038/ki.2011.423

Avolio, A. P., Deng, F. Q., Li, W. Q., Luo, Y. F., Huang, Z. D., Xing, L. F., et al. (1985). Effects of ageing on arterial distensibility in populations with high and low prevalence of hypertension: comparison between urban and rural communities in China. Circulation 71, 202-210. doi: 10.1161/01.CIR.71.2.202

Burt, V. L., Whelton, P., Roccella, E. J., Brown, C., Cutler, J. A., Higgins, M., et al. (1995). Prevalence of hypertension in the US adult population. Results from the Third National Health and Nutrition Examination Survey, 1988-1991. Hypertension 25, 305-313. doi: 10.1161/01.HYP.25.3.305

Cecelja, M., and Chowienczyk, P. (2012). Role of arterial stiffness in cardiovascular disease. JRSM Cardiovasc. Dis. 1:cvd.2012.012016. doi: 10.1258/cvd.2012.012016

Chae, C. U., Pfeffer, M. A., Glynn, R. J., Mitchell, G. F., Taylor, J. O., and Hennekens, C. H. (1999). Increased pulse pressure and risk of heart failure in the elderly. JAMA 281, 634-639. doi: 10.1001/jama.281.7.634

Cohn, J. N., Quyyumi, A. A., Hollenberg, N. K., and Jamerson, K. A. (2004). Surrogate markers for cardiovascular disease: functional markers. Circulation 109(Suppl 1), 31-34. doi: 10.1161/01.CIR.0000133442.99186.39
Our findings support that aortic stiffening (increased PWV and AI) may be an early manifestation of aging. More research is required to investigate how Klotho interacts with the mechanisms of arterial stiffness in old swine. These studies serve as fundamental reference for the swine translational model in understanding human disease and testing of potential therapeutics.

\section{DATA AVAILABILITY STATEMENT}

The raw data supporting the conclusions of this article are available on request to the corresponding author.

\section{ETHICS STATEMENT}

The animal study was reviewed and approved by Institutional Animal Care and Use Committee at California Medical Innovations Institute, San Diego.

\section{AUTHOR CONTRIBUTIONS}

XG conducted the experiments. XG collected and analyzed the data. XG and GK designed the experiments and revised the manuscript.

\section{FUNDING}

This research was funded in part by 3DT Holdings. GK is the founder of 3DT Holdings.

Cruickshank, K., Riste, L., Anderson, S. G., Wright, J. S., Dunn, G., and Gosling, R. G. (2002). Aortic pulse-wave velocity and its relationship to mortality in diabetes and glucose intolerance: an integrated index of vascular function? Circulation 106, 2085-2090. doi: 10.1161/01.CIR.0000033824. 02722.F7

Csiszar, A., Tarantini, S., Fülöp, G. A., Kiss, T., Valcarcel-Ares, M. N., Galvan, V., et al. (2017). Hypertension impairs neurovascular coupling and promotes microvascular injury: role in exacerbation of Alzheimer's disease. Geroscience 39, 359-372. doi: 10.1007/s11357-017-9991-9

Dalton, G. D., Xie, J., An, S., and Huang, C. (2017). New insights into the mechanism of action of soluble Klotho. Front. Endocrinol. 8:323. doi: 10.3389/ fendo.2017.00323

Dernellis, J., and Panaretou, M. (2005). Aortic stiffness is an independent predictor of progression to hypertension in nonhypertensive subjects. Hypertension 45, 426-431. doi: 10.1161/01.HYP.0000157818.58878.93

Franklin, S. S., Khan, S. A., Wong, N. D., Larson, M. G., and Levy, D. (1999). Is pulse pressure useful in predicting risk of coronary heart disease? Circulation 100, 354-360. doi: 10.1161/01.CIR.100.4.354

Greenwald, S. (2007). Ageing of the conduit arteries. J. Pathol. 211, 157-172. doi: $10.1002 /$ path.2101

Guo, X., Lu, X., Yang, J., and Kassab, G. S. (2014). Increased aortic stiffness elevates pulse and mean pressure and compromises endothelial function in Wistar rats. Am. J. Physiol. Heart Circ. Physiol. 307, H880-H887. doi: 10.1152/ ajpheart.00265.2014

Hu, M. C., Shiizaki, K., Kuro-o, M., and Moe, O. W. (2013). Fibroblast growth factor 23 and Klotho: physiology and pathophysiology of an endocrine 
network of mineral metabolism. Annu. Rev. Physiol. 75, 503-533. doi: 10.1146/ annurev-physiol-030212-183727

Hu, M. C., Shi, M., Zhang, J., Quiñones, H., Kuro-o, M., and Moe, O. W. (2010). Klotho deficiency is an early biomarker of renal ischemia-reperfusion injury and its replacement is protective. Kidney Int. 78, 1240-1251. doi: $10.1038 / \mathrm{ki} .2010 .328$

Imura, A., Iwano, A., Tohyama, O., Tsuji, Y., Nozaki, K., Hashimoto, N., et al. (2004). Secreted Klotho protein in sera and CSF: implication for posttranslational cleavage in release of Klotho protein from cell membrane. FEBS Lett. 565, 143-147. doi: 10.1016/j.febslet.2004.03.090

Izzo, J. L. Jr. (2004). Arterial stiffness and the systolic hypertension syndrome. Curr. Opin. Cardiol. 19, 341-352. doi: 10.1097/01.hco.0000126581.89648.10

James, M. A., Watt, P. A., Potter, J. F., Thurston, H., and Swales, J. D. (1995). Pulse pressure and resistance artery structure in the elderly. Hypertension 26, 301-306. doi: 10.1161/01.HYP.26.2.301

Justice, J. N., Ferrucci, L., Newman, A. B., Aroda, V. R., Bahnson, J. L., Divers, J., et al. (2018). A framework for selection of blood-based biomarkers for geroscience-guided clinical trials: report from the TAME Biomarkers Workgroup. Geroscience 40, 419-436. doi: 10.1007/s11357-018-0042-y

Kelly, R. P., Hayward, C., Avolio, A. P., and O’Rourke, M. F. (1989). Noninvasive determination of age-related changes in the human arterial pulse. Circulation 80, 1652-1659. doi: 10.1161/01.CIR.80.6.1652

Koyama, D., Sato, Y., Aizawa, M., Maki, T., Kurosawa, M., Kuro-o, M., et al. (2015). Soluble $\alpha$ Klotho as a candidate for the biomarker of aging. Biochem. Biophys. Res. Commun. 467, 1019-1025. doi: 10.1016/j. bbrc. 2015.10 .018

Kuro-o, M., Matsumura, Y., Aizawa, H., Kawaguchi, H., Suga, T., Utsugi, T., et al. (1997). Mutation of the mouse Klotho gene leads to a syndrome resembling ageing. Nature 390, 45-51. doi: 10.1038/36285

Kurosu, H., Yamamoto, M., Clark, J. D., Pastor, J. V., Nandi, A., Gurnani, P., et al. (2005). Suppression of aging in mice by the hormone Klotho. Science 308, 1829-1833. doi: 10.1126/science.1112766

Laurent, S., Katsahian, S., Fassot, C., Tropeano, A. I., Gautier, I., Laloux, B., et al. (2003). Aortic stiffness is an independent predictor of fatal stroke in essential hypertension. Stroke 34, 1203-1206. doi: 10.1161/01. STR.0000065428.03209.64

Levy, B. I., Ambrosio, G., Pries, A. R., and Struijker-Boudier, H. A. (2001). Microcirculation in hypertension: a new target for treatment? Circulation 104, 735-740. doi: 10.1161/hc3101.091158

Liao, D., Wong, T. Y., Klein, R., Jones, D., Hubbard, L., and Sharrett, A. R. (2004). Relationship between carotid artery stiffness and retinal arteriolar narrowing in healthy middle-aged persons. Stroke 35, 837-842. doi: 10.1161/01. STR.0000120310.43457.AD

Lim, K., Lu, T. S., Molostvov, G., Lee, C., Lam, F. T., Zehnder, D., et al. (2012). Vascular Klotho deficiency potentiates the development of human artery calcification and mediates resistance to fibroblast growth factor 23. Circulation 125, 2243-2255. doi: 10.1161/CIRCULATIONAHA. 111.053405

Lu, X., Guo, X., Wassall, C. D., Kemple, M. D., Unthank, J. L., and Kassab, G. S. (2011). Reactive oxygen species cause endothelial dysfunction in chronic flow overload. J. Appl. Physiol. 110, 520-527. doi: 10.1152/ japplphysiol.00786.2009

Mattace-Raso, F. U., van der Cammen, T. J., Hofman, A., van Popele, N. M., Bos, M. L., Schalekamp, M. A., et al. (2006). Arterial stiffness and risk of coronary heart disease and stroke: the Rotterdam Study. Circulation 113, 657-663. doi: 10.1161/CIRCULATIONAHA.105.555235

Merz, A. A., and Cheng, S. (2016). Sex differences in cardiovascular ageing. Heart 102, 825-831. doi: 10.1136/heartjnl-2015-308769

Mitchell, G. F., Guo, C. Y., Benjamin, E. J., Larson, M. G., Keyes, M. J., Vita, J. A., et al. (2007). Cross-sectional correlates of increased aortic stiffness in the community: the Framingham Heart Study. Circulation 115, 2628-2636. doi: 10.1161/CIRCULATIONAHA.106.667733

Mitchell, G. F., Parise, H., Benjamin, E. J., Larson, M. G., Keyes, M. J., Vita, J. A., et al. (2004). Changes in arterial stiffness and wave reflection with advancing age in healthy men and women: the Framingham Heart Study. Hypertension 43, 1239-1245. doi: 10.1161/01.HYP.0000128420.01881.aa

Mitchell, G. F., Vita, J. A., Larson, M. G., Parise, H., Keyes, M. J., Warner, E., et al. (2005). Cross-sectional relations of peripheral microvascular function, cardiovascular disease risk factors, and aortic stiffness: the Framingham
Heart Study. Circulation 112, 3722-3728. doi: 10.1161/CIRCULATIONAHA. 105.551168

Moe, S. M., and Drueke, T. (2008). Improving global outcomes in mineral and bone disorders. Clin. J. Am. Soc. Nephrol. 3, S127-S130. doi: 10.2215/ CJN.04331206

Paula, R. S., Souza, V. C., Machado-Silva, W., Almeida, B. R., Daros, A. C., Gomes, L., et al. (2016). Serum Klotho (but not haplotypes) associate with the post-myocardial infarction status of older adults. Clinics 71, 725-732. doi: 10.6061/clinics/2016(12)09

Puddu, P., Puddu, G. M., Zaca, F., and Muscari, A. (2000). Endothelial dysfunction in hypertension. Acta Cardiol. 55, 221-232. doi: 10.2143/AC.55. 4.2005744

Richter, B., Haller, J., Haffner, D., and Leifheit-Nestler, M. (2016). Klotho modulates FGF23-mediated NO synthesis and oxidative stress in human coronary artery endothelial cells. Pflugers Arch. 468, 1621-1635. doi: 10.1007/ s00424-016-1858-x

Rodgers, J. L., Jones, J., Bolleddu, S. I., Vanthenapalli, S., Rodgers, L. E., Shah, K., et al. (2019). Cardiovascular risks associated with gender and aging. J. Cardiovasc. Dev. Dis. 6:19. doi: 10.3390/jcdd6020019

Safar, M., Chamiot-Clerc, P., Dagher, G., and Renaud, J. F. (2001). Pulse pressure, endothelium function, and arterial stiffness in spontaneously hypertensive rats. Hypertension 38, 1416-1421. doi: 10.1161/hy1201.096538

Safar, M. E., and London, G. M. (2000). Therapeutic studies and arterial stiffness in hypertension: recommendations of the European Society of Hypertension. The Clinical Committee of Arterial Structure and Function. Working Group on Vascular Structure and Function of the European Society of Hypertension. J. Hypertens. 18, 1527-1535. doi: 10.1097/00004872-200018110-00001

Saito, Y., Nakamura, T., Ohyama, Y., Suzuki, T., Iida, A., Shiraki-Iida, T., et al. (2000). In vivo Klotho gene delivery protects against endothelial dysfunction in multiple risk factor syndrome. Biochem. Biophys. Res. Commun. 276, 767-772. doi: 10.1006/bbrc.2000.3470

Saito, Y., Yamagishi, T., Nakamura, T., Ohyama, Y., Aizawa, H., Suga, T., et al. (1998). Klotho protein protects against endothelial dysfunction. Biochem. Biophys. Res. Commun. 248, 324-329. doi: 10.1006/bbrc.1998.8943

Semba, R. D., Cappola, A. R., Sun, K., Bandinelli, S., Dalal, M., Crasto, C., et al. (2011). Plasma Klotho and mortality risk in older community-dwelling adults. J. Gerontol. A Biol. Sci. Med. Sci. 66, 794-800. doi: 10.1093/gerona/ glr058

Shiraki-Iida, T., Aizawa, H., Matsumura, Y., Sekine, S., Iida, A., Anazawa, H., et al. (1998). Structure of the mouse Klotho gene and its two transcripts encoding membrane and secreted protein. FEBS Lett. 424, 6-10. doi: 10.1016/ S0014-5793(98)00127-6

Swindle, M. M., and Smith, A. C. (1998). Comparative anatomy and physiology of the pig. Scand. J. Lab. Anim. Sci. 25(Suppl 1), 1-10.

Takenaka, T., Kobori, H., Inoue, T., Miyazaki, T., Suzuki, H., Nishiyama, A., et al. (2017). Klotho supplementation attenuates blood pressure and oxidative stress in diabetes. J. Hypertens. 35:e38. doi: 10.1097/01.hjh.0000523076.42214.98

Tarantini, S., Giles, C. B., Wren, J. D., Ashpole, N. M., Valcarcel-Ares, M. N., Wei, J. Y., et al. (2016). IGF-1 deficiency in a critical period early in life influences the vascular aging phenotype in mice by altering miRNA mediated post-transcriptional gene regulation: implications for the developmental origins of health and disease hypothesis. Age 38, 239-258. doi: 10.1007/ s11357-016-9943-9

Toth, P., Tarantini, S., Csiszar, A., and Ungvari, Z. (2017). Functional vascular contributions to cognitive impairment and dementia mechanisms and consequences of cerebral autoregulatory dysfunction, endothelial impairment, and neurovascular uncoupling in aging. Am. J. Physiol. Heart Circ. Physiol. 312, H1-H20. doi: 10.1152/ajpheart.00581.2016

van der Heijden-Spek, J. J., Staessen, J. A., Fagard, R. H., Hoeks, A. P., Boudier, H. A., and Van Bortel, L. M. (2000). Effect of age on brachial artery wall properties differs from the aorta and is gender dependent: a population study. Hypertension 35, 637-642. doi: 10.1161/01.HYP.35.2.637

Vlachopoulos, C., Aznaouridis, K., and Stefanadis, C. (2010). Prediction of cardiovascular events and all-cause mortality with arterial stiffness: a systematic review and meta-analysis. J. Am. Coll. Cardiol. 55, 1318-1327. doi: 10.1016/j. jacc.2009.10.061

Xiao, N. M., Zhang, Y. M., Zheng, Q., and Gu, J. (2004). Klotho is a serum factor related to human aging. Chin. Med. J. 117, 742-747. doi: 10.3760/ cma.j.issn.0366-6999.2004.05.123 
Yamamoto, M., Clark, J. D., Pastor, J. V., Gurnani, P., Nandi, A., Kurosu, H., et al. (2005). Regulation of oxidative stress by the anti-aging hormone Klotho. J. Biol. Chem. 280, 38029-38034. doi: 10.1074/jbc.M509039200 Yamazaki, Y., Imura, A., Urakawa, I., Shimada, T., Murakami, J., Aono, Y., et al. (2010). Establishment of sandwich ELISA for soluble alpha-Klotho measurement: age-dependent change of soluble alpha-Klotho levels in healthy subjects. Biochem. Biophys. Res. Commun. 398, 513-518. doi: 10.1016/j.bbrc.2010.06.110

Conflict of Interest: GK is the founder of 3DT Holdings. GK had the involvement with the study design and preparation of the manuscript.
The remaining author declares that the research was conducted in the absence of any commercial or financial relationships that could be construed as a potential conflict of interest.

Copyright $\odot 2020$ Guo and Kassab. This is an open-access article distributed under the terms of the Creative Commons Attribution License (CC BY). The use, distribution or reproduction in other forums is permitted, provided the original author(s) and the copyright owner(s) are credited and that the original publication in this journal is cited, in accordance with accepted academic practice. No use, distribution or reproduction is permitted which does not comply with these terms. 